\title{
Frequency Selective Surfaces as Near Infrared Electro-Magnetic Filters for Thermophotovoltaic Spectral Control
}

Ryan T. Kristensen, John F. Beausang and David M. DePoy

This report was prepared as an account of work sponsored by the United States Government. Neither the United States, nor the United States Department of Energy, nor any of their employees, nor any of their contractors, subcontractors, or their employees, makes any warranty, express or implied, or assumes any legal liability or responsibility for the accuracy, completeness or usefulness of any information, apparatus, product or process disclosed, or represents that its use would not infringe privately owned rights. 


\title{
Frequency Selective Surfaces as Near Infrared Electro-
}

\section{Magnetic Filters for Thermophotovoltaic Spectral Control}

\author{
Ryan T. Kristensen, John F, Beausang, and David M. DePoy
}

Jockheed Martin Corporation, 2401 River Rd, Schenectady, NY 12309

\begin{abstract}
Frequency selective surfaces (FSS) effectively filter electromagnetic radiation in the microwave band $(1 \mathrm{~mm}$ to $100 \mathrm{~mm})$. Interest exists in extending this technology to the near infrared $(1 \mu \mathrm{m}$ to $10 \mu \mathrm{m})$ for use as a filter of thermal radiation in thermophotovoltaic (TPV) direct energy conversion. This paper assesses the ability of FSS to meet the strict spectral performance requirements of a TPV system. Inherent parasitic absorption, which is the result of the induced currents in the FSS metallization, is identified as a significant obstacle to achieving high spectral performance.
\end{abstract}

040.5350 Photovoltaic, 040.3060 Infrared, 120.2440 Filters, 350.2450 Filters, absorption, 120.5700 Reflection, 120.7000 Transmission, 230.3990 Microstructure devices, 310.3840 Materials and process characterization

\section{Introduction}

Frequency selective surfaces (FSS) selectively reflect and transmit incident electromagnetic radiation via currents induced in a periodic array of metal It has been proposed that an FSS with sub-micron feature size would be a suitable filter for thermal radiation $(1-10 \mu \mathrm{m})$ in a thermophotovoltaic (TPV) direct energy conversion application., ${ }^{2,4}$ The filter is a key component in TPV because only a fraction (typically less 
than $30 \%$ ) of the incident thermal radiation has energy exceeding the diode bandgap energy, $\mathrm{E}_{\mathrm{g}}$, and can thus be converted to electric power. Consequently, a high-efficiency, high-power density TPV application ${ }^{5}$ relies upon both a high performing photovoltaic diode and a high performing filter; unlike solar photovoltaics, which only require a good diode and a relatively simple anti-reflection coating.

The two purposes of a TPV filter are (1) to minimize parasitic heat transfer by reflecting below. bandgap radiation back to the radiator, and (2) to maximize transmission of the useful above-bandgap energy to the diode. TPV spectral control performance is therefore characterized by two key metrics: filter efficiency and integrated above-bandgap transmission. The filter efficiency, $\eta_{\text {filter }}$, is a direct multiplier on system efficiency $\left(\eta_{\mathrm{TPV}}=\eta_{\text {diode }} \cdot \eta_{\text {filter }}\right)$. Filter above-bandgap transmissivity, $\mathrm{T}_{\text {filter }}$, must be integrated over angle and wavelength and is proportional to system power density. The highest performing non-FSS TPV filter technology to date is comprised of a tandem configuration of an optical interference filter (dielectric stack) mounted on a plasma filter (heavily doped semiconductor). The tandem filter achieves measured spectral efficiencies of approximately $80 \%$ and a transmissivity of approximately $80 \%$ with diode bandgap equal to $0.52 \mathrm{eV}$ (or, equivalently, $\lambda_{\mathrm{Eg}}=2.4 \mu \mathrm{m}$ ) and radiator temperature equal to $954^{\circ} \mathrm{C}$. Our program has been considering FSS as an alternate filter technology to replace or augment the current tandem filter. As will be discussed in this paper, intrinsic absorption in the pass-band of a metallic $\mathrm{FSS}^{7,8}$ is very likely to preclude its use as a filter in a high efficiency, high power density TPV system

FSS was initially considered as a candidate for TPV spectral control based upon its performance in the millimeter wavelength and the potential for high below-bandgap reflectivity due to the high carrier concentration in a metal. The approach of this paper is first to explain the TPV spectral control performance metrics of filter efficiency and integrated above-bandgap transmissivity. Spectral performance of a fabricated and a modeled FSS structure is then shown to be significantly lower than a TPV interference/plasma tandem filter. The poor relative performance of FSS is predominately due to both high above-bandgap reflection, which lowers the transmissivity, and absorption in the FSS pass 
band, which lowers both transmissivity and filter efficiency. Thirdly, the mechanism for absorption is explained and different strategies to mitigate it are explored, see Table It is concluded that even if the above-bandgap reflectivity could be reduced, the inherent ohmic losses in a metallic FSS, which are the source of the prohibitively high absorption, limit the use of FSS as a high-efficiency TPV filter. In this study we did not consider the additional FSS challenges of reducing the high above-bandgap reflectivity and ensuring operation over the full angular dispersion.

\section{TPV Spectral Control}

\section{Theory}

Excellent spectral control is required for a high efficiency TPV system because of the unfavorable fraction of above-bandgap energy $\left(\mathrm{E}>\mathrm{E}_{\mathrm{g}}\right.$ or $\left.\lambda<\lambda_{\mathrm{Eg}}\right)$ compared to below-bandgap $\left(\mathrm{E}<\mathrm{E}_{\mathrm{g}}\right.$ or $\left.\lambda>\lambda_{\mathrm{Eg}}\right)$ energy radiated from a blackbody source. For example, only $26 \%$ of the energy radiated by a $950^{\circ} \mathrm{C}$ blackbody would exceed a $0.52 \mathrm{eV}$ diode bandgap. An ideal TPV filter would reflect all incident below-bandgap radiation energy back to the radiator and reflect none of the above-bandgap energy, thus transmitting the useful energy to the diode. Slight deviations from $100 \%$ below-bandgap reflectivity can result in significant parasitic heat transfer, and hence low system efficiency, while increased above-bandgap reflectivity reduces transmissivity (i.e., system power density). Above-bandgap parasitic absorption reduces both metrics, while below-bandgap parasitic absorption only reduces filter efficiency.

Filter efficiency depends predominantly on the radiator temperature, diode bandgap, and below. bandgap reflectivity and is defined as the amount of transmitted above-bandgap energy relative to the total amount of absorbed energy:

$$
\eta_{\text {filter }}=\frac{\int_{0}^{2 \pi} \int_{0}^{\frac{\pi}{2}} \int_{0}^{\lambda_{\mathrm{Eg}}} T_{\text {filter }}(\lambda, \theta, \phi) N\left(\lambda, T_{\text {rad }}\right) \sin (\theta) \cos (\theta) d \lambda d \theta d \phi}{\iint_{0}^{\frac{2 \pi}{2}} \int\left(1-R_{\text {filter }}(\lambda, \theta, \phi)\right) N\left(\lambda, T_{\text {rad }}\right) \sin (\theta) \cos (\theta) d \lambda d \theta d \phi}
$$


where $T_{\text {filter }}$ is the transmission fraction, $R_{\text {filter }}$ is the reflection fraction, $N$ is Planck blackbody energy spectral density (W/ $\mathrm{cm}^{2}-\mu \mathrm{m}$-steradian), $\lambda_{\mathrm{Eg}}$ is the bandgap wavelength $(\mu \mathrm{m}), \lambda$ is the radiation wavelength $(\mu \mathrm{m}), T_{\text {rad }}$ is the radiator temperature, $\theta$ is the polar angle of incidence (normal to the FSS plane), and $\phi$ is the azimuthal angle of incidence.

Note that equation (1) is integrated over all angles and assumes a blackbody radiator, thereby incorporating the large amount of energy at relatively long wavelengths and also the isotropic nature of blackbody radiation. Unlike solar photovoltaics, which have only one angle of incident flux, the relatively small separation between the diode and radiator result in inherently Lambertian TPV illumination. Consequently, TPV filters are extremely challenging as they must operate over a large spectral range $(\sim 10 \mu \mathrm{m})$ and all angles of incidence.

\section{TPV Tandem Filter}

We estimate the achievable limits of TPV spectral control (which depend on diode bandgap and radiator temperature) to be a filter efficiency of $85-90 \%$ and an integrated above-bandgap transmission of approximately $85 \%$. Figure 1 shows the measured spectral response (reflection, $R(\lambda)$; transmission, $T(\lambda)$; absorption, $\mathrm{A}(\lambda)$ ) of a state-of-the-art TPV interference/plasma tandem filter for a $0.52 \mathrm{eV}$ device. The filter in Figure is designed for an incident angle of $45^{\circ}$, since this is the peak angle from a Lambertian Its performance, however, will vary with angle of incidence, and therefore the reflectivity in Figure is portrayed by an angle-of-incidence weighted value. The transmission is measured at normal incidence, and the absorption determined by subtracting it and the reflectivity at $11^{\circ}$ from unity $(\mathrm{A}=1$ -

Ideally, the reflectivity would also be measured at zero degrees, but the smallest measurable angle in the experimental setup is $11^{\circ}$. The dielectric-stack interference filter provides a sharp turn-on between high transmission of above-bandgap radiation to high reflection for below-bandgap radiation. For practical reasons, however, the interference filter does not reflect the full range of below-bandgap photons, thus requiring a plasma filter ${ }^{9}$ to reflect the lowest energy $(\lambda>6 \mu \mathrm{m})$ radiation. The interference 
filter enhances the performance of the plasma filter by sharpening its slow reflection turn-on and covering; the elevated absorption at the plasma frequency. The interference/plasma filter performance metrics of filter efficiency $\left(\eta_{\text {filter }}\right)$, integrated above-bandgap transmission $\left(T_{\mathrm{E}>\mathrm{Eg}}\right)$, integrated below-bandgap reflectivity $\left(\mathrm{R}_{\mathrm{E}<\mathrm{Eg}}\right)$, integrated above-bandgap reflectivity $\left(\mathrm{R}_{\mathrm{E}>\mathrm{Eg}}\right)$, integrated above-bandgap absorption $\left(\mathrm{A}_{\mathrm{E}>\mathrm{Eg}}\right)$ are shown in Table for a $954^{\circ} \mathrm{C}$ radiator temperature and $0.52 \mathrm{eV}$ diode bandgap.

In principle, below-bandgap reflection can be increased to compensate for slightly larger amounts of above-bandgap absorption because filter efficiehcy represents the integrated heat transfer across the entire spectrum. In FSS filter design it will be shown that such a trade-off is difficult to achieve because the techniques that increase below-bandgap reflectivity (e.g., more metal coverage, dielectric layers, or multiple FSS layers) simultaneously increase the parasitic absorption.

For computational reasons, FSS structures are modeled only at normal incidence at the most favorable azimuthal $(\phi)$ orientation for a given FSS geometry. Variation in reflectivity with polar $(\theta)$ angle-of-incidence is common for any filter, but variation with azimuthal angle is specific to FSS because of the 2D periodicity of the FSS array and element shape. Fabricated structures were measured at numerous orientations (both $\theta$ and $\phi$ ), but for consistency with the modeling, the performance metrics were calculated near normal incidence. The degradation in FSS spectral performance for off-design angles is ignored here, but must be considered in actual TPV applications as the decrement is considerable and unavoidable due to the Lambertian distribution of the radiation. Consequently, the performance metrics in Table are optimistic predictions of FSS performance. An idealized $45^{\circ}$-only spectral performance is also calculated for the interference/plasma tandem filter for better comparison to the FSS structures.

\section{FSS filter Design}

FSS selectively reflect and transmit incident radiation depending on the superposition between the incident field and the scattered field from the induced currents in the metallic FSS. Transparency is not 
the result of the thinness of the metallization, as the FSS is opaque to the relevant wavelengths. The induced currents are determined by the geometry of the FSS and can be represented by a resonant circuit of inductive, capacitive, and resistive elements. ${ }^{1}$ The resistance is due to the finite conductivity of the metal structure, and results in ohmic losses that are measured as absorption in the FSS structure. An FSS modeled as a perfect electrical conductor does not show any absorption. This absorption mechanism is thus intrinsic to FSS operation given the finite conductivity of the metal, and is particularly detrimental to TPV filter performance because the peak absorption occurs at or near the peak in filter transmission. Physically, the peak absorption is a maximum at the filter resonance because the induced currents reach peak values and the maximum fraction of the FSS metal (i.e. both sides of the filter) participates in the resonant behavior.

FSS resonant frequency and spectral response is determined by the precise dimensions of the exact FSS geometry including element size, shape, width, pitch, thickness, and spacing. Thin ( 100's $\mathrm{nm}$ ) dielectric layers on either side of the FSS, including the substrate, are important design features that affect the location of the resonance and magnitude of the absorption by altering the electromagnetic boundary condition on the metal surface. Different conductivity metals and the specific frequencydependence of the conductivity will also impact the resonance, especially for TPV applications in the near-infrared frequency regime. ${ }^{7}$ Lastly, multiple FSS layers can also be stacked in an attempt to achieve the desired spectral response.

\section{Fabrication and Modeling}

As a result of the complexities of even a simple FSS structure, numerical codes are essential for determining the spectral response. FSS structures were modeled using Ansoft's finite-element High Frequency Structure Simulator (HFSS) code. A TEM radiation field was specified at normal incidence to the FSS and oriented parallel to the symmetry planes of the FSS array. The response was determined by modeling the smallest symmetric unit cell of the FSS geometry with periodic boundary conditions. Dielectric layer and metal dimensions were modeled consistent with fabricated structures, while the 
substrate was assumed to be semi-infinite. Frequency-dependent optical properties of metal were obtained from handbook values, ${ }^{6}$ while the dielectric layer properties were assumed frequencyindependent and non-absorbing. The power in the reflected and transmitted fields was calculated and subtracted from unity to determine the absorption. The calculation was repeated with adaptive size wavelength intervals between 1 and $10 \mu \mathrm{m}$. After benchmarking to simple non-FSS structures that could be solved analytically, HFSS was used to duplicate the spectral performance of fabricated FSS structures. Good agreement was achieved and confirmed the origin of the absorption as the ohmic losses of the induced currents within the FSS metal. Finally, the code was used to interrogate alternate FSS structures in order to minimize absorption.

Ring-aperture aluminum FSS structures with sub-micron feature size, Figure 2, are fabricated via phase-shift lithography at MIT Lincoln Laboratory ${ }^{10}$ on double-side polished silicon substrates. Prior to FSS fabrication, $350 \mathrm{~nm}$ of $\mathrm{SiO}_{2}$ is grown on the front and back of the wafer via thermal oxidation. The front dielectric layer is incorporated into the FSS design, while the back layer serves as a simple antireflection coating for measurement purposes. Several FSS configurations were explored including various FSS geometries (rings and wire mesh), dimensions, dielectric layers, and metallizations. Additional $\mathrm{SiO}_{2}$ layers were grown on top of the FSS via plasma-enhanced chemical vapor deposition. The specular reflection and transmission of all filters were measured at Lockheed Martin with a Nicolet Magna 750 FTIR, referenced to a reflecting gold standard.

\section{Absorption in FSS}

'igure 3 illustrates a comparison between fabricated and modeled FSS structures. The fabricated structure is a hexagonal array of circular ring apertures with dimensions indicated in Figure 2, but is modeled in HFSS as a rectangular array to minimize computation. The effect of this assumption on absorption is minimal considering the similar metallization area fractions for hexagonal $(0.633)$ and rectangular (0.682) unit cells. The resonant frequency and bandwidth, however, will be slightly different from the actual hexagonal case due to the small change in inter-element spacing. The $\mathrm{SiO}_{2}$ layers and 
Silicon substrate are treated as non-absorbing dielectrics with constant relative permittivity $\varepsilon_{\mathrm{r}, \mathrm{SiO} 2}=2.25$ and $\varepsilon_{\mathrm{r}, \mathrm{Si}}=56$, respectively. By assuming non-absorbing dielectrics the absorption is confined entirely to the ohmic losses in the metallic FSS.

In general, good agreement is achieved between measured data and simulated results. The slight discrepancy in resonant frequency may be attributed to the assumption of a rectangular versus hexagonal array, uncertainty in fabricated versus modeled dimensions, $11^{\circ}$ measurement instead of normal incidence as modeled, or the assumption of wavelength-independent, non-absorbing optical properties for the dielectric. The large absorption peak at $9.5 \mu \mathrm{m}$ in the fabricated FSS is due to $\mathrm{Si}-\mathrm{O}$ bond absorption in the $\mathrm{SiO}_{2}$, and is not included in the modeled $\mathrm{SiO}_{2}$ or the spectral efficiency calculations of measured data. The spectral performance is compared to the tandem filter in Table The low performance characteristics are expected considering the relatively slow reflection turn-on of the FSS at the bandedge and the high above-bandgap absorption. The spectral performance of the fabricated FSS is optimal for a diode with a lower bandgap $\left(\mathrm{E}_{\mathrm{g}}=0.45 \mathrm{eV}\right)$ than the tandem filter $(0.52 \mathrm{eV})$. Consequently, the calculated parameters in the table are determined at this lower bandgap for a best-case-scenario comparison. In general, there is a strong trade-off between transmissivity, filter efficiency, diode bandgap, and radiator temperature. In Table we report values for a $954^{\circ} \mathrm{C}$ radiator and a diode bandgap that provides high filter efficiency near the peak of integrated above-bandgap transmission.

The absorption in the pass-band of the FSS is seen to be significantly higher than a tandem filter. The high above-bandgap reflectivity, which reduces the above-bandgap transmissivity, is also of concern. Next, several FSS-design techniques to mitigate the absorption are explored including: reducing abovebandgap absorption by (1) eliminating the dielectric, (2) replacing the aluminum metal with silver, and (3) reducing the metal area with a wire-mesh design. The below-bandgap reflectivity of the wire mesh is then increased by considering (4) a double-layer wire mesh FSS and (5) an interference filter mounted on the single-layer wire mesh FSS. It will be shown that the only approach that significantly improves the spectral performance is the addition of an interference filter to the wire mesh FSS, which despite the 
aggressive assumptions remains below the interference/plasma tandem filter performance. All of the other cases considered had either significant above-bandgap absorption or low below-bandgap reflectivity and hence relatively poor spectral performance.

\section{Alternate FSS Designs}

\section{Free-standing ring-aperture FSS}

The same rectangular array ring-aperture geometry of Figure 2 is simulated as a free-standing structure in vacuum (no dielectric layers and no substrate) to assess the impact of the dielectric on absorption. Figure 4 indicates that the peak absorption decreased from $\sim 19 \%$ in the dielectric FSS (Figure 3 ) to $\sim 13 \%$ in the free-standing FSS. While conceptually interesting, 13\% absorption remains prohibitively large for most TPV applications, and is physically unrealistic as the FSS must be deposited on some kind of substrate. Additionally, the dielectric layers also serve an important role in FSS filter design by increasing the filter transmission bandwidth and shifting the resonance ${ }^{1}$ to the desired frequency. Consequently, removing the dielectric is seen to shift the resonance to shorter wavelengths and the narrower pass-band decreases the integrated transmissivity. Spectral performance for the free-standing FSS is determined in Table

It is interesting to note that the increased FSS absorption due to the adjacent dielectric layers is due to the electromagnetic boundary condition at the metal-dielectric interface, and not specific to the FSS geometry. The effect is illustrated by considering the absorption in a metal plate due to incident electromagnetic radiation from an arbitrary dielectric medium. Since the power absorbed in the metal plate will be due to the ohmic losses of the induced surface current density, the ratio of power absorbed to incident upon the plate, $A$, can be shown to be proportional to the index of refraction of the medium:

$$
A=\frac{4 R_{s}(\lambda)}{\eta_{o}} \sqrt{\varepsilon_{r}}=\frac{4 R_{s}(\lambda)}{\eta_{o}} n
$$

Where $n$ is the index of refraction of the dielectric medium, $\eta_{0}$ is the intrinsic impedance of free space (377 $\Omega$ ) and $R_{s}$ is the surface resistance of the metal plate $(\Omega /$ square). The absorptivity of the plate (or an 
FSS) therefore scales directly with the index of refraction of the surrounding medium. The wavelengthdependent nature of the resistance is expressed explicitly to emphasize the importance of using appropriate material properties when considering infrared applications.

\section{Increase DC conductivity of Metal}

FSS absorption can also be reduced by decreasing the resistance of the metal, which reduces the ohmic losses experienced by induced currents. In the free-standing ring-aperture FSS model the complex permittivity of aluminum is replaced with silver, which at $6.17 \times 10^{7} \mathrm{~S} / \mathrm{m}$ has the highest room temperature DC (zero frequency) conductivity of any metal, and is almost twice the $3.54 \times 10^{7} \mathrm{~S} / \mathrm{m}$ DC conductivity of aluminum. Figure 5 shows that the spectral performance of a silver ring-aperture FSS compared to aluminum (the FSS structure is the same as shown in Figure 2, but without the silicon substrate). The performance of the two is similar with a small shift in the silver FSS resonant frequency to slightly longer wavelengths because of the lower optical constants of silver relative to aluminum. The DC conductivity is not a good FSS metric in the near-infrared where the AC conductivity of aluminum is actually higher than silver as evidenced by comparing the material optical constants ${ }^{6}\left(\sigma_{\mathrm{AC}}=2 n k \omega \varepsilon_{0}\right.$ where the symbols have their usual meanings with $k=$ extinction coefficient). Therefore, altering the metallization of the FSS to another highly conducting metal does not significantly improve the absorption and thus will not improve the spectral performance of the FSS.

\section{Single-layer wire mesh FSS}

The ohmic losses encountered in the ring-aperture FSS can be reduced by minimizing the surface area of metallization with a wire mesh FSS geometry. Since the wire mesh consists of less metal, which is the source of the ohmic loss, there may be less absorption. Two other factors, however, prevent significant improvement. First, is that the lower fraction of metal coverage results in lower reflectivity of belowbandgap radiation, which reduces filter efficiency. Secondly, the FSS dielectric layers require a greater 
dielectric constant in order to increase the inherently low capacitance of the widely-spaced elements in a wire mesh geometry, ${ }^{1}$ thus increasing absorption hear the resonance for the reason discussed in case 1.

The modeled wire mesh FSS is therefore embedded in a more-optimal dielectric $\left(\varepsilon_{\mathrm{r}}=3.6\right.$ versus $\varepsilon_{\mathrm{r}}=2.25$ of $\mathrm{SiO}_{2}$ ). The predicted spectral performance and the FSS dimensions are shown Figure 6, and indicate a peak absorption of approximately $14 \%$ with less integrated above-bandgap absorption compared to the dielectric ring-aperture (Figure 3) and the free-standing ring-aperture FSS (Figure 4), see Table The decrease in above-bandgap absorption does not significantly improve spectral performance because of the relatively large decrement in below-bandgap reflectivity of a wire mesh.

These modeling trends were confirmed in a fabricated gold wire mesh structure by MIT Lincoln Laboratory, see Figure 7. The fabricated sample, however, differs from the modeled structure in that the grid spacing is almost twice as large, there is no layer of dielectric on top of the FSS, and the dielectric layer is $\mathrm{SiO}_{2}$, not the optimized dielectric with $\varepsilon_{\mathrm{r}}=3.6$. Consequently, the location of the measured resonance (Figure 8$)$ is shifted to shorter wavelengths $(1.8 \mu \mathrm{m})$, and the magnitude of its peak absorption $(\sim 6 \%)$ is lower. Despite the improvement in reduced above-bandgap absorption for the measured and modeled wire mesh FSS compared to the ring-aperture structures, see Table 1, both the modeled and fabricated wire mesh FSS have low below-bandgap reflectivity, and therefore poor filter efficiency.

Figure 9 illustrates the shift in spectral response of an FSS due to variable angle illumination. Normal incidence is compared to $\theta=45^{\circ}$ angle-of-incidence with the same azimuthal orientation $\left(\phi=0^{\circ}\right)$, and to $\theta=45^{\circ}$ angle-of-incidence illumination with $\phi=45^{\circ}$ azimuthal orientation. Actual FSS TPV filters would need to consider these dependencies in an appropriately angle-weighted (both angles) performance. Except for the measured interference/plasma tandem filter, these dependencies were not included in either the modeling or the measured results of Table

\section{Double-layer wire mesh FSS}


In order to increase the below-bandgap reflectivity of the wire mesh design, a second FSS layer is stacked on the first. The second FSS wire mesh is aligned directly on top of the first, with the same aluminum metal dimensions and dielectric as the FSS modeled in case 3. The spectral results of the double-layer wire mesh structure are shown in Figure 10. The below-bandgap reflection has increased as desired; however, the above-bandgap absorption has almost doubled, see Table As might be expected, doubling the FSS structure has resulted in a doubling of absorption. It is thus concluded that mitigating absorption with multiple FSS layers is not feasible for a high efficiency TPV filter. Double layer FSS structures were not fabricated.

\section{FSS-Interference filter combination}

The below-bandgap reflectivity can also be increased by including an interference filter on top of the FSS filter to improve the sharpness of the reflectivity turn-on. The spectral performance, Figure 1 is assumed by superimposing the interference filter spectral performance and measured FSS performance The estimated spectral performance of the gold wire mesh FSS of Figure 7 with an interference filter is shown in Figure Spectral performance is improved, see Table 1, but the optimistic performance estimation is still not comparable to the interference/plasma tandem filter performance. Also realize that the assumption of no $\theta$ and no $\phi$ angular degradation is aggressive, further reducing the likelihood of a successful FSS filter concept in a high-efficiency TPV application.

\section{Conclusion}

All FSSs fabricated to date by MIT Lincoln Laboratory and modeled by Lockheed Martin exhibit prohibitively high absorption in the filter pass-band except for a wire mesh geometry, which had low below-bandgap reflectivity. The spectral response of fabricated FSS structures has been successfully modeled with a commercial finite element software code, and good agreement with measured results obtained. he origin of the absorption is attributed to ohmic losses in the FSS metallization, and is believed to be inherent-even in a perfectly fabricated structure - as it is the result of the induced currents 
in the finite conductivity FSS metal. Several strategies to mitigate the absorption were explored, but absorption was reduced only at the expense of reducing below-bandgap reflectivity. This negative tradeoff prevents any gains in filter efficiency. If it is assumed that FSS filters could be optimized for isotropic infrared radiation and the above-bandgap reflectivity successfully reduced, the significant absorption in the pass band remains a fundamental obstacle to incorporating FSS into high-efficiency, high-power density TPV spectral control. It is thus concluded that FSS with conventional metallizations (those other than superconducting materials, which were not considered) do not satisfy the strict requirements for high spectral efficiency as compared to current interference/plasma tandem filter technology.

\section{Acknowledgements}

The authors would like to thank to Ted Lyszczarz, David Astolfi, Steven Spector, Sandy Deneault, Donna Lennon, and Carl Creel at MIT Lincoln Laboratory for fabricating the FSS structures.

\section{References}

B. Munk, Frequency Selective Surfaces, (John Wiley \& Sons, Inc, New York, 2000).

2 T. K. Wu, "Infrared Filters for High Efficiency Thermophotovoltaic Devices," Microwave and Optical Tech. Let. 15, pp. 9-12 (1997).

3 W. E. Horne, M. D. Morgan, and V. S. Sundaram, "IR Filters for TPV Converter Modules," in AIP Conf. Proceedings \#358, The Second NREL Conference on Thermophotovoltaic Generation of Electricity, J. P. Benner, T.J. Coutts, and D. S. Ginley, eds. (AIP Press, Woodbury, NY, 1996), pp. $35-51$

4 W. E. Horne and M. D. Morgan, "Filter Array for Modifying Radiant Thermal Energy," U.S. Patent 5,611,870, issued March 18, 1997.

5 R. R. Siergiej, B. Wernsman, S. A. Derry, R. G. Mahorter, R. J. Wehrer, S. D. Link, M. N. Palmisiano, R. L. Messham, S. Murray, C. S. Murray, F. Newman, J. Hills, and D. Taylor, “ $20 \%$ Efficient InGaAs/InPAs Thermophotovoltaic Cells," in AIP Conf. Proceedings \#653, The Fifth 
NREL Conference on Thermophotovoltaic Generation of Electricity, J. J. Coutts, G. Guazzoni, J Luther, eds. (AIP Press, Melville, NY, 2003), pp. 414-423.

6. R. C. Weast, CRC Handbook of Chemistry and Physics $64^{\text {th }}$ ed, (CRC Press, Boca Raton, 1983). E. Tobsakal and J. L. Volakis, "On the Properties of Materials for Designing Filters at Optical Frequencies," in Proceedings of the 2003 IEEE AP-S International Symposium and URSI National Radio Science Meeting, 4:635-638, June 2003

8. J.B. Pryor, "On Ohmic Losses in Frequency|Selective Surfaces at Near-Infrared Wavelengths," (Ph.D. Thesis, Ohio State University, Department of Electrical Engineering, 2003, unpublished).

9. G.W. Charache, D.M. DePoy, J.E. Raynolds, P.F. Baldasaro, K.E. Miyano, T. Holden, F. H. Pollack, P.R. Sharps, M.L. Timmons, C.B. Geller, W. Mannstadt, R. Asahi, A.J. Freeman, W. Wolf, "Moss-Burstein and Plasma Reflection Characteristics of Heavily-Doped N-type $\operatorname{In}_{\mathrm{x}} \mathrm{Ga}_{1-\mathrm{x}} \mathrm{As}$ and InP $\mathrm{yAs}_{1-\mathrm{y}}$, , J. Appl. Phys, 86, pp. 452-462, (1999).

10. S. J. Spector, D. K. Astolfi, S. P. Doran, T. M. Lyszczarz and J. E. Raynolds, "Infrared Frequency Selective Surfaces Fabricated Using Optical Lithography and Phase Shift Masks," J. Vac. Sci.

Technol. B 19, pp. 2757-2760, (2001 


\section{Figure Captions}

Figure Measured TPV tandem filter reflection (angle-of-incidence weighted) and absorption (A = 1-R$\mathrm{T})$ as a function of wavelength. For clarity, transmission data at $11^{\circ}$ is not shown.

Figure 2 SEM (gray is metal and black is dielectric) and schematic showing fabricated dimensions of hexagonal-array aluminum ring-aperture FSS

3 Spectral performance of aluminum ring-aperture FSS modeled at normal incidence (line) and measured at $11^{\circ}$ angle-of-incidence (points).

Figure 4 Predicted spectral performance of free-standing aluminum ring-aperture FSS in vacuum at normal incidence.

5 Predicted spectral performance of silver (dotted) and aluminum (solid) ring-aperture FSS.

Figure 6 Predicted spectral performance of aluminum wire mesh FSS at normal incidence.

Figure 7 SEM (gray is metal and black is dielectric) and schematic showing fabricated dimensions of gold wire mesh FSS.

Figure 8 Measured spectral response of fabricated gold wire mesh FSS at $11^{\circ}$ incidence angle.

Figure 9 Measured reflectivity of fabricated gold wire mesh FSS at (1) $\theta=0^{\circ}$ and $\phi=0^{\circ}$ (pluses), (2) $\theta=$ $45^{\circ}$ and $\phi=0^{\circ}$ (triangles), and (3) $\theta=45^{\circ}$ and $\phi=45^{\circ}\left(\mathrm{x}^{\prime} \mathrm{s}\right)$.

Figure 10 Predicted spectral response of a double-layered aluminum wire mesh FSS at normal incidence.

Figure Estimated spectral performance of single wire mesh gold FSS with interference filter.

\section{List of Tables}

able Comparison of Tandem and FSS Filter performance ${ }^{\dagger}$ 


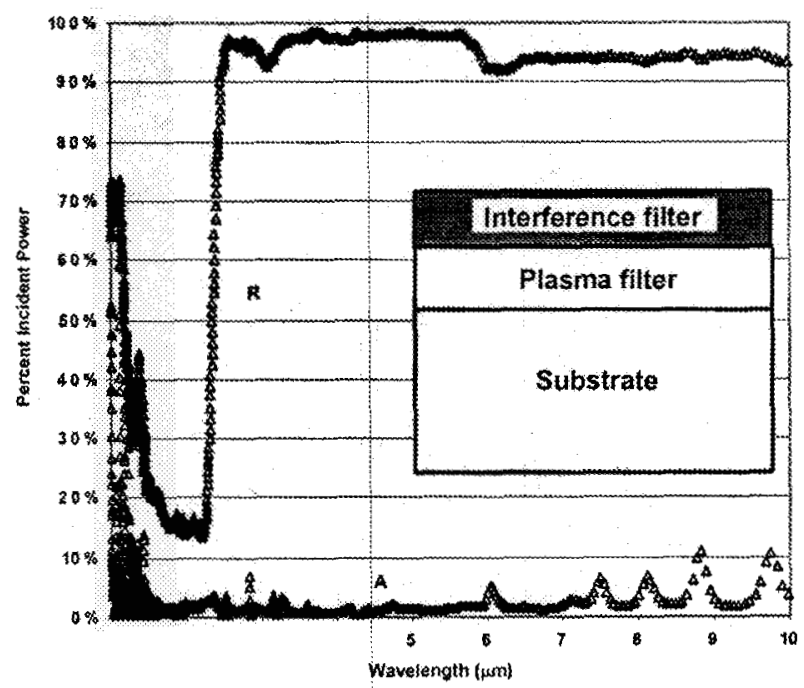

Figure 1 Measured TPV tandem filter reflection (angle-of-incidence weighted) and absorption (A = 1-R-T) as a function of wavelength. 


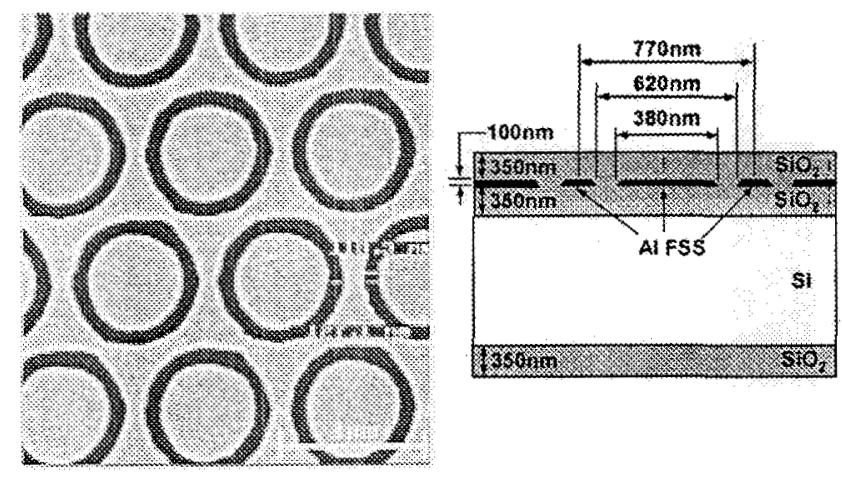

Figure 2 SEM (gray is metal and black is dielectric) and schematic showing fabricated dimensions of hexagonal-array aluminum ring-aperture FSS 


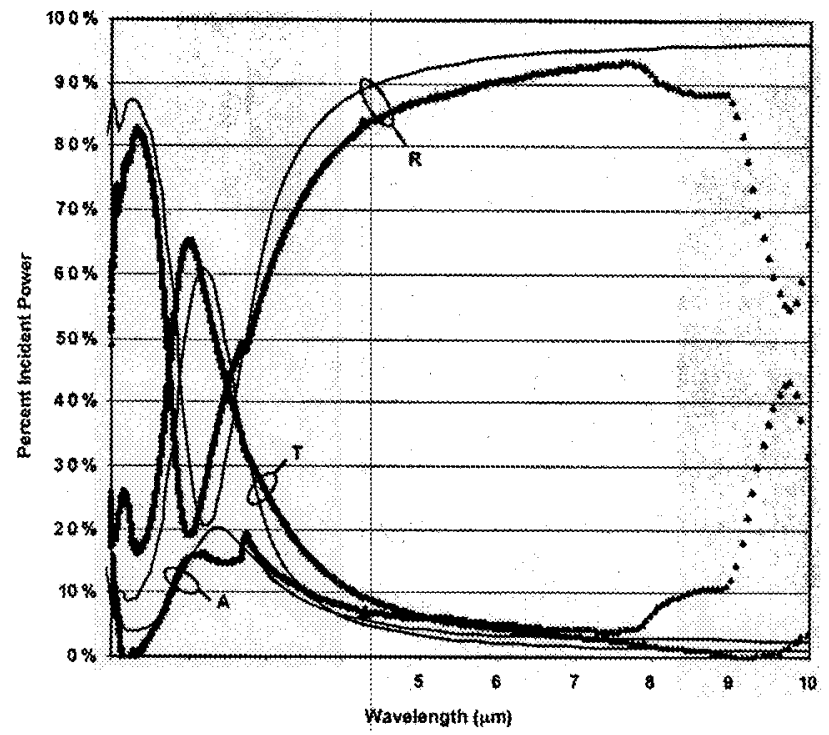

Figure 3 Spectral performance of aluminum ring-aperture FSS modeled at normal incidence (line) and measured at $11^{\circ}$ angle-of-incidence (points). 


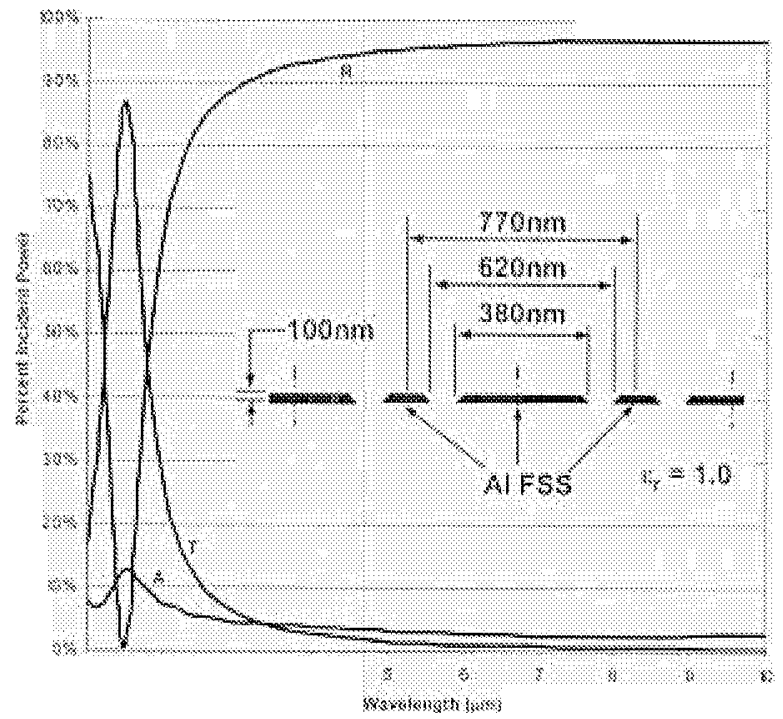

Figure 4 Predicted spectral performance of free-standing aluminum ring-aperture FSS in vacuum at normal incidence. 


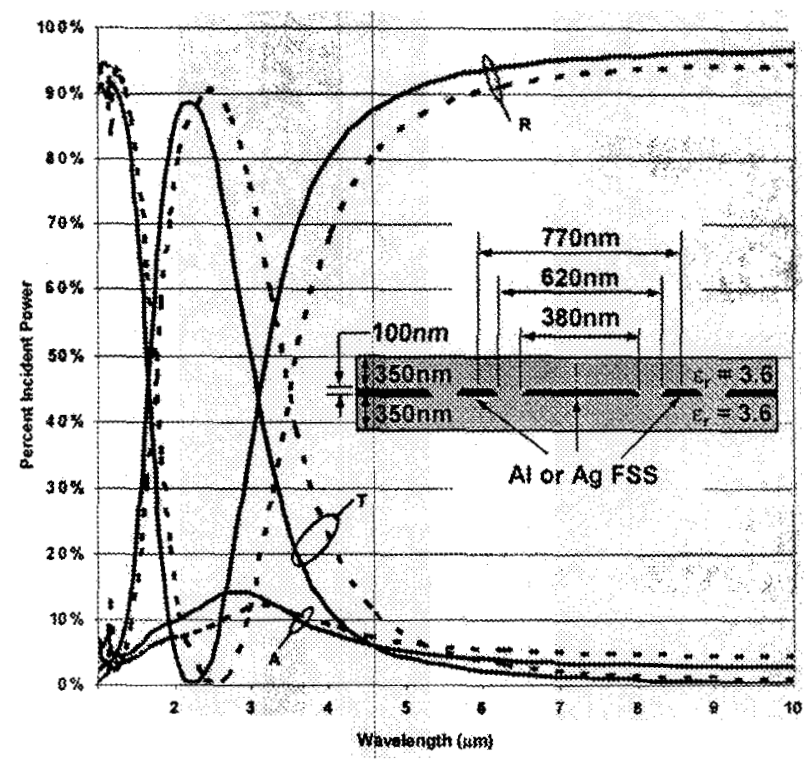

Figure 5 Predicted spectral performance of silver (dotted) and aluminum (solid) ring-aperture FSS. 


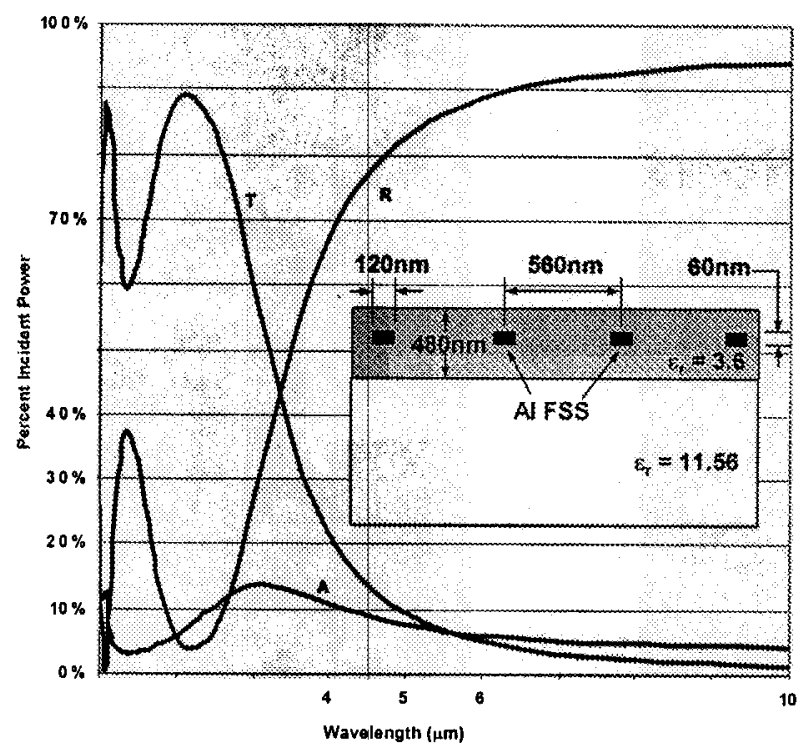

Figure 6 Predicted spectral performance of aluminum wire mesh FSS at normal incidence. 

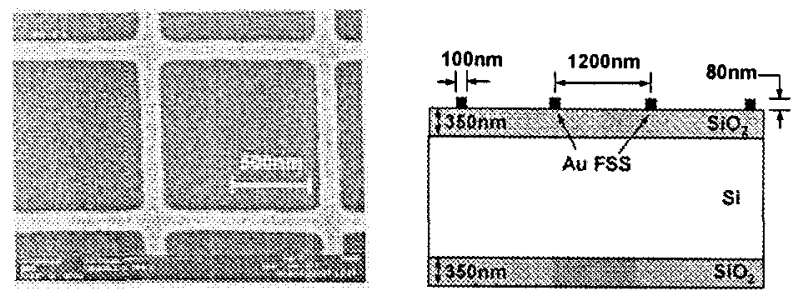

Figure 7 SEM (gray is metal and black is dielectric) and schematic showing fabricated dimensions of gold wire mesh FSS 


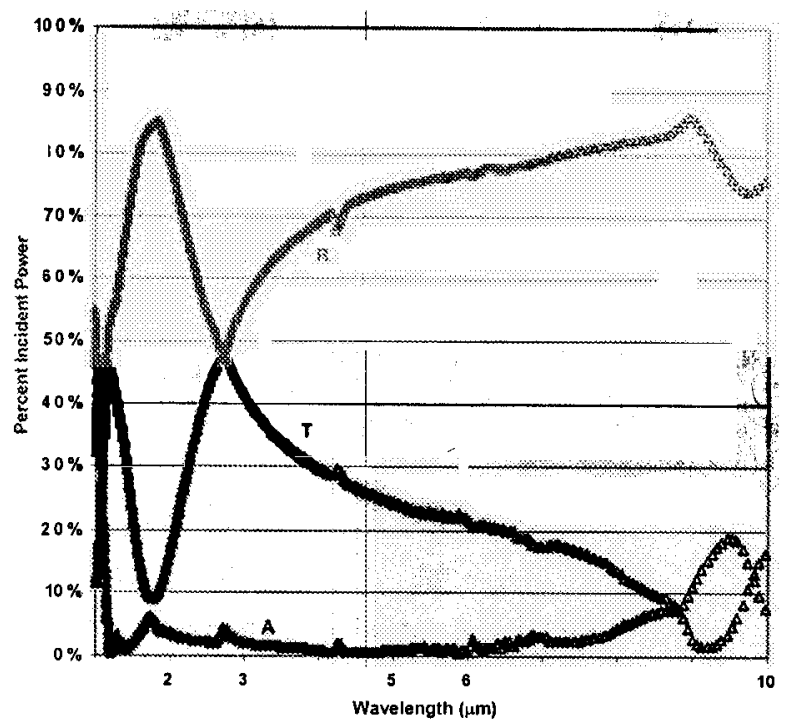

Figure 8 Measured spectral response of fabricated gold wire mesh FSS at $11^{\circ}$ incidence 


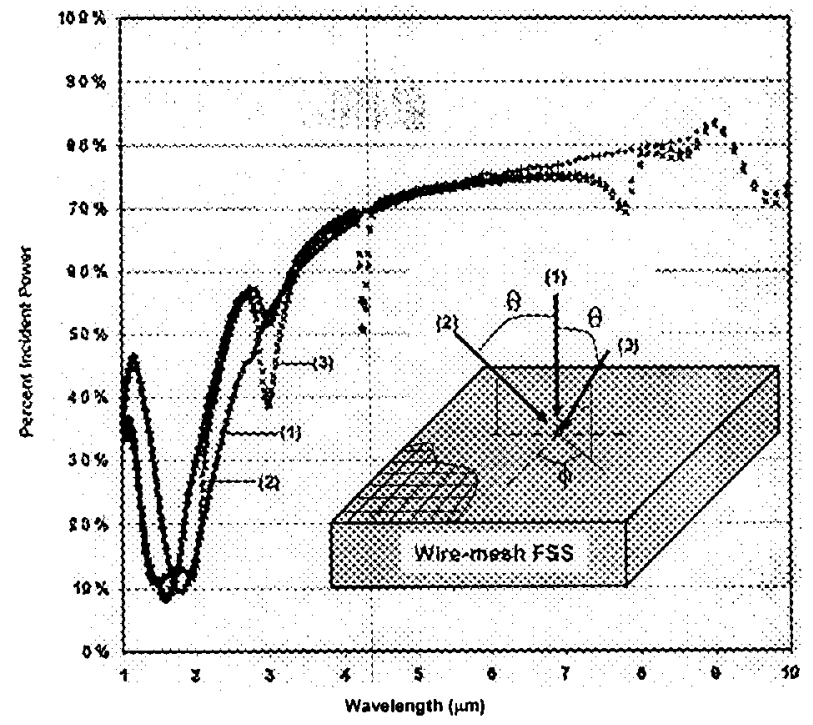

Figure 9 Measured reflectivity of fabricated gold wire mesh FSS at (1) $\theta=0^{\circ}$ and $\phi=0^{\circ}$ (pluses), (2) $\theta=45^{\circ}$ and $\phi=0^{\circ}$ (triangles), and (3) $\theta=45^{\circ}$ and $\phi=45^{\circ}$ ( $x^{\prime} s$ ). 


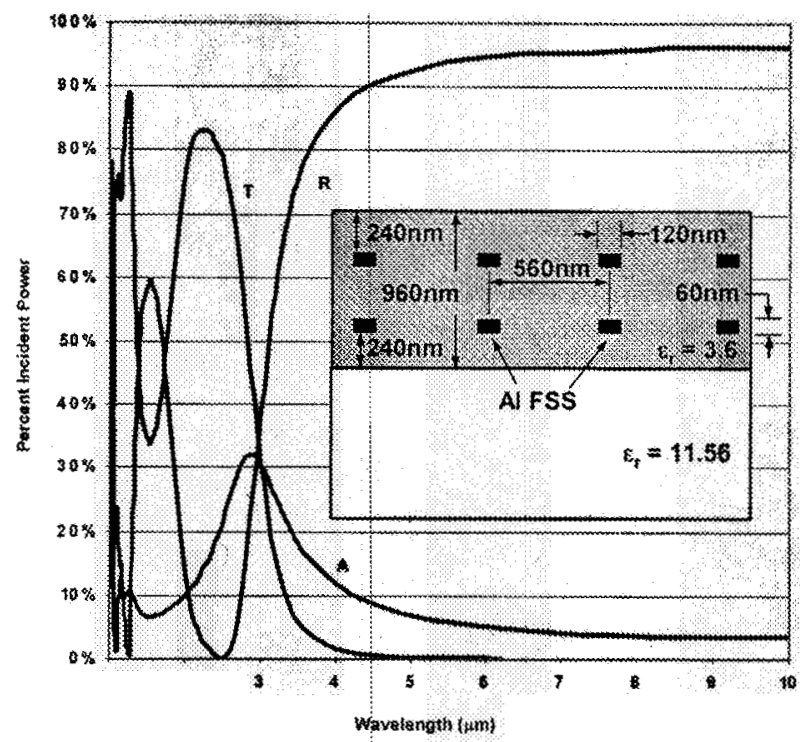

Figure 10 Predicted spectral response of a double-layered aluminum wire mesh FSS at normal incidence. 


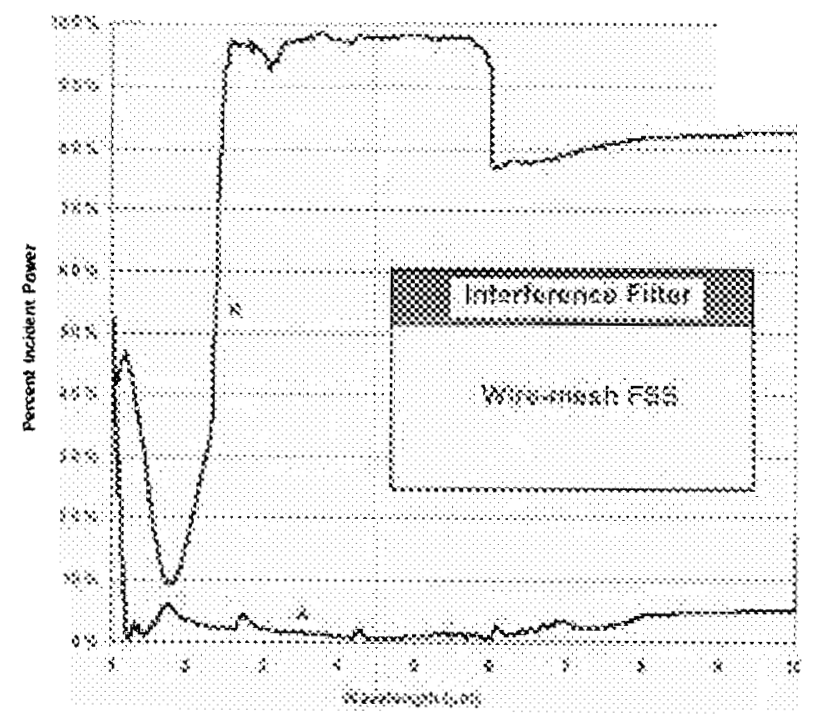

Figure 11 Estimated spectral performance of single wire mesh gold FSS with interference filter. 
Table 1 Comparison of Tandem and FSS Filter performance $\dagger$

\begin{tabular}{|c|c|c|c|c|c|}
\hline 8. $840 \%$ & 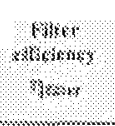 & 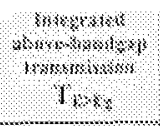 & 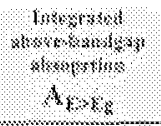 & 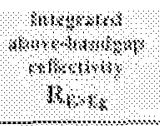 & 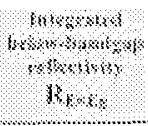 \\
\hline $\begin{array}{l}\text { TPV Filter } \\
\text { Goul }\end{array}$ & $85-90 \%$ & $85 \%$ & $3 \%$ & $15 \%$ & $97 \%$ \\
\hline $\begin{array}{l}\text { Tanden filter* } \\
\text { (measured) }\end{array}$ & $80 \%$ & $80 \%$ & $3 \%$ & $18 \%$ & $94 \%$ \\
\hline $\begin{array}{l}\text { Tandem filter** } \\
\text { (calculated) }\end{array}$ & $85 \%$ & $84 \%$ & $3 \%$ & $13 \%$ & $96 \%$ \\
\hline $\begin{array}{l}\text { Ring-aperture FSs } \\
\text { (measured) }\end{array}$ & $-48 \%$ & $\sim 45 \%$ & $\sim 12 \%$ & $\sim 43 \%$ & $\sim 80 \%$ \\
\hline $\begin{array}{l}\text { Ring-aperture FSS } \\
\text { (calculated) }\end{array}$ & $\sim \mathbf{5 0 \%}$ & $\sim 40 \%$ & $\sim 14 \%$ & $\sim 461 / 4$ & $-85 \%$ \\
\hline $\begin{array}{l}\text { Free-standing } \\
\text { Ring-aperture FSs' } \\
\text { (calculated) }\end{array}$ & 55\% & $\sim 45 \%$ & $\sim 10 \%$ & $\sim 45 \%$ & $\sim 92 \%$ \\
\hline $\begin{array}{l}\text { Wire-mesh FSS } \\
\text { (calculated) }\end{array}$ & $\sim 55 \%$ & $\sim 80 \%$ & $\sim 7 \%$ & $\sim 13 \%$ & $-68 \%$ \\
\hline $\begin{array}{l}\text { Wire-mesh FSS } \\
\text { (measuredl) }\end{array}$ & $\sim 45 \%$ & $\sim 71 \%$ & $\sim 5 \%$ & $\sim 24 \%$ & $\sim 67 \%$ \\
\hline $\begin{array}{l}\text { Double-layer } \\
\text { Wire-mesth FSS } \\
\text { (calculated) }\end{array}$ & $-56 \%$ & $\sim 68 \%$ & $\sim 14 \%$ & $\sim 18 \%$ & $\sim 80 \%$ \\
\hline $\begin{array}{l}\text { Wirt-mesh FSS } \\
\text { w linter f(frence } \\
\text { Filter } \\
\text { Filestimated) }\end{array}$ & $\sim 75 \%$ & $-66 \%$ & $-5 \%$ & $\sim 29 \%$ & $\sim 93 \%$ \\
\hline $\begin{array}{l}\dagger \text { Parameters ca } \\
* \quad \text { Angle-of-inci } \\
* * 45^{\circ} \text { Angle-of- } \\
\ddagger \quad 0^{\circ} \text { Angle-of- } \\
\$ 11^{\circ} \text { Angle-of- }\end{array}$ & $\begin{array}{l}\text { lated for } \\
\text { nce weight } \\
\text { cidence ref } \\
\text { cidence ref } \\
\text { cidence ref }\end{array}$ & $\begin{array}{l}\text { timal diode bar } \\
\text { d reflectivity } \\
\text { ctivity only } \\
\text { ctivity only } \\
\text { ctivity only }\end{array}$ & ap $(0.45-0.5$ & and radiato & perature T \\
\hline
\end{tabular}

